Review Article

\title{
Are Civilisation Diseases Chronic Silent Inflammations?
}

\author{
Doepp, Manfred MD,
}

Head of HolisticCenter, 13 Haupt St., Abtwil 9030, Switzerland

www.drdoepp.org holisticcenter1@yahoo.de

\begin{abstract}
The causes of chronic civilisation diseases are still unclear. This article examines and supports the hypothesis that chronic silent inflammation is likely to be the underlying cause. Reactions of the immune system that are excessive and can no longer be controlled by the organism are involved. Triggers can be pathogens, but then the processes continue as autoaggressions. A number of natural remedies are presented that are more than symptomatically effective.
\end{abstract}

The ever-increasing so-called diseases of civilisation, such as diabetes mellitus, dementia, Alzheimer's, Parkinson's, multiple sclerosis and many others, have neither a known cause nor a cause-related therapy. Symptoms are tinkered with and patients are told that unfortunately there is no effective treatment or even a cure yet. Accordingly, selftreatment groups are formed, which give the impression that one can do something after all. Unfortunately, these are sponsored by the pharmaceutical companies and thus make dependent, so that no new paths are taken.

However, unnoticed findings are accumulating that these diseases are based on one principle: it is the chronic, smouldering inflammation that is not caused by pathogens. Their mechanisms have actually been researched, but there are no consequences. This is because chemical pharmaceuticals are difficult to use.

It is known which endogenous molecules are involved: tumour necrosis factor $\dot{\alpha}$, interleukins, cytokines and prostaglandins. The association with "tumour" is misleading, it has little to do with tumours.

They are cytokines that are mainly secreted by macrophages (phagocytes). Let's look up TNF on Wikipedia (figure 1):

"The regulation of all these steps is extremely complex. Overall, TNF is an extremely versatile cytokine with very many functions in different contexts. TNF shares many of its functions with interleukin-1 $\beta$ and interleukin-6. TNF does the following in the various organ systems:

- In the hypothalamus:

$o$ Increased release of $\mathrm{CRH}$ and via this stimulation of the hypothalamic-pituitary-adrenal axis.

o Suppression of appetite.

- In the liver: formation of acute phase proteins such as CrP.

- In macrophages: stimulation of phagocytosis.

- On neutrophil granulocytes, TNF has a migratory effect.

- In all tissues: It leads to increased insulin resistance and an increase in the activity of cyclooxygenase-2.

A locally increased concentration of TNF leads to the classic symptoms of inflammation: heat, swelling, redness and pain. TNF- $\alpha$ inhibiting drugs are mainly used in rheumatism therapy." 


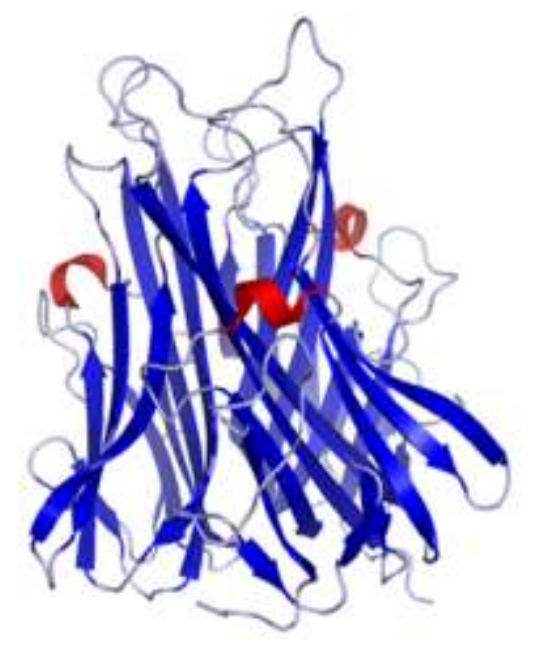

Figure 1: TNF-ó, a highly complex protein

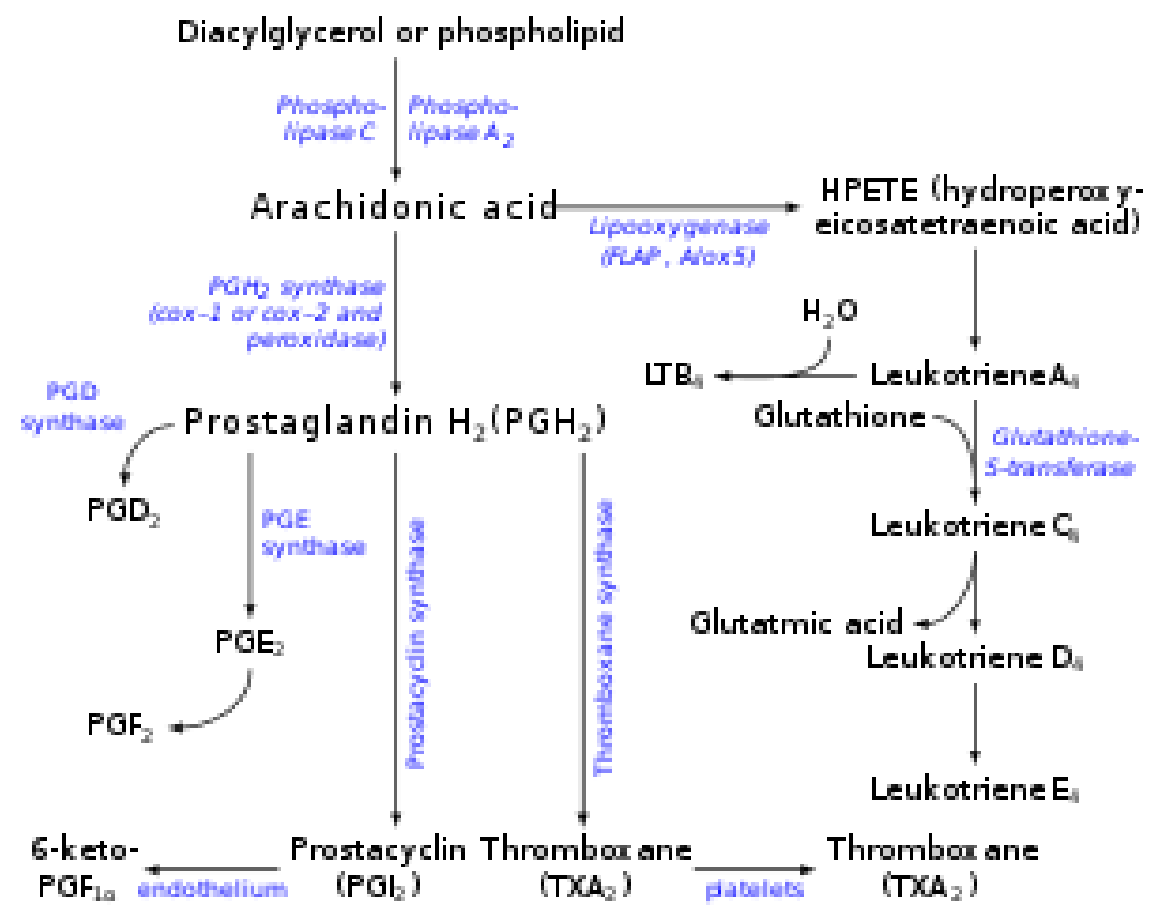

Figure 2 : Biosynthesis of Prostaglandins

In summary, it can be said that it is a non-specific but very effective stress reaction that puts the affected tissue into a state of alarm, an overreaction often without any real meaning. From a laboratory point of view, it is above all an increased $\mathrm{CrP}$ that catches the eye. But more important are the interleukins.

Wikipedia: "Dysregulation at any level of interleukin-1 $\beta$ activation and secretion can trigger IL-1 mediated disease...ILI $\beta$ is a stimulator of neurons secreting corticotropin-releasing hormones in the hypothalamus, and via them leads to stimulation of ACTH and cortisone secretion in the adrenal glands during inflammatory reactions. . $I L 1 \beta$, like interleukin-1 $\alpha$, acts on two interleukin-1 receptors: the interleukin-1 receptor type 1 (ILIRI) carries the signals of IL1, it is always present on all cells, and its expression is hardly regulated."

Prostaglandin E2 is also involved. Wikipedia: "PGE2, together with PGI2, is the main prostaglandin involved in inflammation. It increases vascular permeability (resulting in tissue swelling), is involved in the development of redness and increases pain (which is caused by other inflammatory substances such as bradykinin or histamine)."

What is known is that hospitalised Covid-19 patients do not die from the viruses, but from the cytokine storm along with blockages of the ACE2 receptors by spike proteins. This storm produces, among other things, a tendency to 


\section{Doepp, Manfred et. /al Are Civilisation Diseases Chronic Silent Inflammations?}

thrombosis with microembolism and multiple organ failure: "consumption coagulopathy". Similarly, deaths after mRNA vaccination can be explained if too few neutralising antibodies for the spike proteins are available.

\section{Consequences?}

What can be concluded from the above? Let us note that during their historical development, higher organisms created a highly reactive system that had and still has the task of reacting immediately to influences/stimuli/potential dangers, stopping normal processes and producing an overreaction, even at the risk of harming themselves. In the early stages of humanity this rarely happened, we all know the situation of confrontation with a sabre-toothed tiger. A balance between non-specific immunological reaction and its attenuation, suppression and termination was easy to establish, although the activating factors were far in the majority. A calm re-emerged on its own in the Stone Age and was the normal state.

Today, however, we are predominantly in a perpetual state of alarm. It has been proven that not only the ringing of a mobile phone, but already the expectation of the possibility of a ringing produces a state of stress in the brain, as it used to correspond to a fight for life and death $(1,2,3,4)$.

So far, so bad. But how can we prove that these reactions cause our diseases of civilisation? Let's take the most important complex, the degenerations in the brain.

Our belly fat forms the primary depot for harmful substances. But unfortunately, secondarily, the brain, where nonspecific inflammatory reactions are the result.

For a long time, science assumed that prions and amyloid plaques were the cause of chronic brain diseases. As was often the case, a coincidence of effects was thought to be the cause.

Only recently have we been surprised to read that a rethink is taking place, see in the Swiss Sunday newspaper: "Smouldering fire in the brain". How would it be if the protein deposits in the brain were the result of chronic inflammation in the brain, and if their causes were listed in this article?

Large-scale attempts to remove the plaques from the brain have failed. Nun studies showed no relationship between mental fitness and the number of typical plaques in the brains. No therapy developed so far for brain diseases showed any significant success. Instead, the researchers had to take note of the fact that there is a completely different relationship between fitness and brain structure, namely with regard to the so-called "microglia": the greater their proportion in the brain, the more demented the person.

So what does this microglia do? It is responsible for chronic inflammation. And accordingly, a relationship was found between taking rheumatism medication and avoiding Alzheimer's disease. Microglia is a cell population of its own and originates from stem cells. It has the task of disposing of protein fragments caused by inflammation and which have not been properly broken down. The more it is activated, the stronger the silent inflammation, a vicious circle develops. And this is apparently responsible for the degeneration.

There are indications that rheumatism drugs are most effective in the treatment of such chronic inflammatory conditions. Logic demands it anyway. Chemical treatment would therefore consist of cortisone preparations or nonsteroidal anti-rheumatic drugs.

\section{Natural agents}

To avoid the side effects of these agents, complementary medicine offers a whole spectrum. Some of them are listed here:

- Frankincense (Boswellia serrata or sacra).

- MSM (methyl sulfonyl methane)

- Green-lipped mussel (Perna canaliculus)

- Devil's claw root extract (Harpagophytum proc.)

- Chilli extract (capsaicin)

- Willow bark extract (salicin)

- Chuchuhuasi bark (Maytenus aelivis)

- Myrrh (Commiphora myrrha)

- Opoponax (Commiphora erythraea var. glabrescens) 


\section{Doepp, Manfred et. /al Are Civilisation Diseases Chronic Silent Inflammations?}

- Curcuma (Turmeric)

- Resveratrol, OPC, Pterostilbene

- Reduced glutathione

- PEApure (palmitoylethanolamide)

- Colloidal Gold (20 ppm)

Those who are Bible-savvy will remember that the newborn Jesus was given frankincense, myrrh and colloidal gold by the holy three magi as gifts of great value. It may also be remembered that for centuries there was a frankincense route leading from South Arabia/Yemen/Wadi Hadramaut/Oman to Rome. Cities along it, such as Petra, became rich, so much did the trade flourish. The Catholic and Orthodox Churches sensibly adopted this custom: incense as an antiautoaggressive agent.

A treatment of our civilisation diseases with these remedies would thus be a real alternative. Frankincense has also made a name for itself in the treatment of brain tumours. It is used in high doses that are well tolerated.

The German Society for Biological Cancer Prevention reports on this:

http://www.biokrebs.de/images/stories/download/Therapie_Infos/Hirntumore.pdf.

\section{Individualisation}

In our HolisticCenter we test the virtual remedies to be sent via quantum teleportation and the remedies to be taken with the help of the information field. In the course of time, a ranking list of the remedies that are most often positive resulted. Besides almost all spices, especially from the Orient and India, frankincense is at the top. We recommend, however, not to use it according to a scheme, but to test the remedies to be taken from the above list concretely: how do body systems react (e.g. HRV, Heart-Rate-Variability) when a remedy is taken in hand? One can also use other responsive systems of the organism such as the meridians or the autonomic nervous system (skin resistance).

If the questioned body system reacts positively, it means "yes", if it reacts negatively, it means "no". In this way, therapies can be optimised, objectified and individualised. The remedies with the highest affirmation are used successfully. Why successful? As the organism itself has made the decision.

\section{References}

1. http://www.3sat.de/mediathek/index.php?display=2\&mode=playset\&obj=35776

2. www.nano.de 3Sat Nano Spezial at 09.05.2013, report of Stefan Ebling and Nadia Salem

3. Prof. Manfred Spitzer, University Hospital Ulm, Germany. Book: „Digitale Demenz“.

4. Dr. Alexander J. Galach, Mediz. PräventionsCentrum Hamburg: MRT and Smart-

Phone: long-term shrinking of the hippocampus, short-term: ringing causes

persistent alertness, even if this is only expected 